\title{
CME Genitourinary medicine (100623): self-assessment questionnaire
}

\author{
Author: Edited by Jan Clarke and Tahseen A Chowdhury
}

SAQs and answers are ONLINE for RCP fellows and collegiate members

The SAQs printed in the CME section can only be answered online to achieve external CPD credits. Any comments should be sent in via email only: clinicalmedicine@rcplondon.ac.uk

\section{Format}

Candidates are asked to choose the best answer from the five possible answers. This best of five format is used in many medical examinations, however the questions are not intended to be representative of those used in the MRCP(UK) Part 1 or Part 2 Written Examinations.

The answering process

1 Go to www.rcplondon.ac.uk/SAQ

2 Log on using your usual RCP username and password

3 Select the relevant CME question paper

4 Answer all 10 questions by selecting the best answer from the options provided

5 Once you have answered all the questions, click on Submit

\section{Registering your external CPD credits}

Carrying out this activity allows you to claim two external CPD credits. These will be automatically transferred to your CPD diary, where you can review the activity and claim your points.

1 A 28-year-old man presented with a three-week history of painful, swollen joints affecting his right knee and left ankle with joint stiffness in the morning. He had noticed a urethral discharge, dysuria and irritation in his eyes.

\section{What is the most likely diagnosis?}
(a) gout
(b) herpes simplex infection
(c) rheumatoid arthritis
(d) septic arthritis
(e) sexually acquired reactive arthritis (SARA)

2 Which of the following statements is true regarding sexually acquired reactive arthritis (SARA)?

(a) Chlamydia trachomatis is the most commonly associated infection and requires antibiotic treatment

(b) disease-modifying anti-rheumatic drugs (DMARDs) should be started immediately

(c) genital symptoms usually start after the arthritis has established

(d) long-term antibiotic therapy is needed to control the arthritis

(e) SARA resolves spontaneously within four weeks in most individuals

3 Which of the following descriptions best defines an HIV indicator condition?
(a) a condition associated with an undiagnosed HIV prevalence of $>0.1 \%$
(b) a condition more prevalent in patients living with HIV
(c) a medical condition arising as a result of immune deficiency
(d) an AIDS-defining condition
(e) conditions which share risk factors for HIV infection

4 Which of the following malignant conditions is NOT considered an HIV indicator condition?
(a) anal cancer
(b) head and neck cancer
(c) melanoma
(d) multicentric Castleman's disease
(e) non-Hodgkin lymphoma

5 Which of the following is the most common complication in cardiovascular syphilis?
(a) aortic stenosis
(b) ascending aortitis
(c) descending aortitis
(d) mitral regurgitation
(e) tricuspid regurgitation

6 To which of the following antibiotics has Treponema pallidum developed resistance?
(a) azithromycin
(b) ceftriaxone
(c) co-trimoxazole
(d) doxycycline
(e) procaine penicillin 
7 With regards to current treatment options for hepatitis $C$ virus in an HIV-positive individuals which of the following is correct?

(a) antiretroviral therapy should only be commenced in individuals with CD4 counts of $<500$ cells $/ \mathrm{mm}^{3}$

(b) drug-drug interactions are no longer an important consideration

(c) interferon-free options are currently available for all

(d) there is currently no guidance to allow the treatment of acute hepatitis $C$ with direct-acting antivirals

(e) treatment success rates are inferior to those of the HIVnegative population

8 Which of the following is correct with regards to sexual transmission of hepatitis C virus (HCV)?

(a) HCV RNA is more frequently detected in the semen of HIVpositive than HIV-negative men infected with HCV

(b) post-exposure prophylaxis in the form of immunoglobulin can be offered to those knowingly exposed to HCV

(c) re-infection with HCV can be screened for with HCV antibody

(d) serodiscordant heterosexual couples are routinely counselled on the importance of condom use to prevent transmission

(e) sexual transmission of HCV has been reported more frequently in HIV-negative than HIV-positive individuals.

9 A 42-year-old male presented with an acute-onset mild macular rash, fevers, night sweats, normal liver function tests, lymphadenopathy, diarrhoea, general malaise and a negative point-of-care HIV rapid test result following a reported recent unprotected sex act with an unknown male partner. The patient had no history of foreign travel and was previously vaccinated against hepatitis B virus.

\section{What is the most likely diagnosis?}
(a) acute hepatitis $C$
(b) acute hepatitis B
(c) acute HIV infection
(d) cytomegalovirus
(e) Epstein-Barr virus

10 What is the most sensitive HIV blood test you could request in a young MSM (men who have sex with men) who reports a recent unprotected sex act with a known HIV-positive partner not on antiretroviral therapy within the last 7 days?
(a) HIV combo fourth generation antigen/antibody test
(b) HIV antibody test with RITA (Recent Infection Treatment Algorithm) incident testing
(c) point-of-care HIV test
(d) western blot
(e) HIV DNA

\section{CME Dermatology SAQ \\ Answers to the CME SAQ published in Clinical Medicine February 2016}

$\begin{array}{cccccccccc}\text { Q1 } & \text { Q2 } & \text { Q3 } & \text { Q4 } & \text { Q5 } & \text { Q6 } & \text { Q7 } & \text { Q8 } & \text { Q9 } & \text { Q10 } \\ \text { (d) } & \text { (d) } & \text { (c) } & \text { (a) } & \text { (e) } & \text { (b) } & \text { (d) } & \text { (b) } & \text { (e) } & \text { (a) }\end{array}$

